\title{
Conference report First World Congress on Psychiatric Genetics
}

Cambridge, 3-5 August 1989

MiCHAEL MORRIS, Research Senior Registrar, Institute of Medical Genetics, University of Wales College of Medicine, Cardiff CF4 4XN; and S. J. ADAMS, Lecturer, Academic Department of Psychiatry, St Bartholomew's Hospital, London EC1A 7BE

This conference was organised by the Biological Psychiatry Group of the College under the auspices of the World Federation of Societies of Biological Psychiatry with Dr T. J. Crow as Chairman of the Organising Committee. The meeting was attended by 270 participants from 25 countries. The 150 oral and poster presentations, many of which reported on the application of recombinant DNA techniques to psychiatry, reflected a growing field of intensive research.

The main focus of the meeting was on the genetics of depression and schizophrenia. It is well-known that a locus for bipolar affective disorder has been implicated on chromosome 11 in one Amish family in a study by Professor Janice Egeland et al. However, re-analysis of the data, updated psychiatric status and extensions of the core pedigree have shown that genetic linkage is not as close as was originally reported. Other centres have not replicated the original finding of linkage to chromosome $11 \mathrm{p} 15$.

Dr Hugh Gurling presented data showing that a schizophrenia susceptibility locus may lie on the proximal long arm of chromosome 5(5q11.2-13.3) in seven British and Icelandic families. However, negative studies from various parts of the world were reported (Scotland, Wales, Ireland, USA, Canada, Italy). Evidence supporting a locus for psychosisin the pseudo-autosomal region of the sex chromosomes was presented by the Northwick Park Hospital group. The D2-dopamine receptor gene has been recently cloned and this gene has been linked to chromosome 11. However, strong evidence against linkage of the D2 gene to schizophrenia was presented in a large Swedish kindred.

The great difficulties of research in psychiatric genetics were debated. Segregation studies have suggested that genetic models for psychiatric disorders such as schizophrenia are complex and the pattern of inheritance is often unknown. Therefore lod scores, which are usually applied to single locus models, must be interpreted cautiously. Other problems are genetic heterogeneity, the presence of phenocopies (non-genetic alterations of the phenotype), diagnostic validity and nonrandom mating (for example, schizophrenic sufferers differentially mate with 'alcoholics'). Yet more problems are reduced penetrance, variable age of onset and environmental factors such as life events.

Many strategies for future research were proposed. There should be a high threshold for definition of a case. More families should be recruited and other areas of the genome should be examined for linkage. New linkage programmes should be developed to allow for non-Mendelian forms of inheritance. In the affected sib-pair method of analysis, for example, no model of inheritance is specified.

Several other neuropsychiatric diseases were discussed. Closer probes than $\mathrm{G} 8$ have been discovered to be linked to Huntington's disease and preliminary reports of the clinical application of such probes in predictive testing were presented by several centres (Baltimore, Cardiff, Manchester). Regarding Tourette syndrome, pedigrees of the disorder from the Middlesex Hospital are consistent with autosomal dominant inheritance and a locus on chromosome 18 is under investigation. St George-Hyslop's finding that the genetic defect causing familial Alzheimer's disease maps on chromosome 21 has been replicated at St Mary's Hospital, London. Mental handicap and the molecular study of a fragile site at Xq27.3 are sources of vigorous investigation. The gene for arylsulfatase A (deficiency of which leads to metachromatic leucodystrophy, a recognised cause of psychosis and mental retardation) has been cloned in Germany and this should facilitate the study of genotype-phenotype relationships. Data on the genetics of narcolepsy showed that it has the tightest HLA linkage of any disease $(99 \%$ of all subjects with narcolepsy and cataplexy are DR 2 positive). There is suggestive evidence that there is linkage between alcoholism and the MNS blood group on chromosome 4. Current research indicates that infantile autism is genetically heterogeneous. Professor Andreas Rett, who described the eponymous Rett's syndrome in 1966, gave an account of the profound mental and physical handicaps associated with this disorder.

An important session was held on the ethical and social implications of molecular and clinical psychiatric genetics. These include problems associated with confidentiality and informed consent. The controversial subject of carrier detection was debated but the possibility of applying presymptomatic and prenatal tests in disorders such as schizophrenia is remote. 